\title{
The Different Clinical Manifestations and Diagnostic Strategies of Isolated Sphenoid Non-Invasive Fungal Sinusitis
}

\author{
Ali Almomen ${ }^{1 *}$, Hussain Al Baharna ${ }^{2}$, Abdullah A AlShakhs ${ }^{3}$, Mohammed A Alfalah ${ }^{3}$, Mohammed AlSaeed ${ }^{3}$, Ali \\ AlTuraifi $^{3}$ and Njood Alaboud ${ }^{3}$ \\ ${ }^{1}$ Consultant Rhinology \& Skull base surgery, King Fahad Specialist Hospital, Kingdom of Saudi Arabia \\ ${ }^{2}$ ENT Consultant, Qatif Central Hospital, Kingdom of Saudi Arabia \\ ${ }^{3}$ Medical Intern, Saudi Program, Kingdom of Saudi Arabia \\ *Corresponding author: Ali Almomen, Consultant Rhinology \& skull base surgery, King Fahad Specialist Hospital, Dammam, Kingdom \\ of Saudi Arabia
}

\begin{abstract}
Objective: To present the different clinical manifestations and diagnostic strategies of isolated sphenoid non-invasive fungal sinusitis (SNIFS) in order to prevent delayed diagnosis and providing early management.

Method: This study is a retrospective clinical study, conducted between January 2008 to November 2019. It was done in the ENT department of two institutes: King Fahad specialist Hospital and Qatif Central Hospital, Saudi Arabia. Only patients with sole involvement of non-invasive fungal sinusitis in the sphenoid sinus were included.

Conclusion: The incidence of SNIFS is rare. However, it's clinically important because untreated SNIFS can cause significant complications. The clinical features of SNIFS are ambiguous and nonspecific which make its diagnosis more difficult. Post-nasal discharge and headache refractory to the medical management is the most common symptom of SNIFS. CT scan is still the cornerstone of radiological diagnosis of SNIFS. Endoscopic sinus surgery is the standard care of management.
\end{abstract}

Keywords: Sphenoid sinus, Fungal ball, allergic fungal sinusitis, non-invasive fungal sinusitis, endoscopy, allergic fungal rhinosinusitis

Abbreviations: SNIFS: Sphenoid Non-Invasive Fungal Sinusitis; SAFRS: Sphenoid Allergic Fungal Rhinosinusitis; ISFB: Isolated Sphenoid Fungal Ball; FB: Fungal Ball; AFRS: Allergic Fungal Rhinosinusitis

\section{Introduction}

Histopathological, fungal rhinosinusitis can be divided into invasive and noninvasive infection. Noninvasive fungal rhinosinusitis is further subdivided into fungal ball (FB) and allergic fungal rhinosinusitis (AFRS) [1, 2]. FB is an accumulation of dense fungal hyphae, most commonly in the maxillary sinus [1]. AFRS is an immunological reaction to fungal antigen rather than a fungal infection. Interestingly, it is common in immunocompetent younger patient with atopic background, with similar findings and presentation as in chronic rhinosinusitis [3]. Sphenoid sinusitis is usually present in concurrent with other sinuses. However, in rare cases isolated sphenoid has been reported, which represent only $2.7 \%$ of all sinus diseases $[3,4]$. There are several important structures adjacent to sphenoid sinus which vulnerable to injury through any sphenoid sinus lesion, including the pituitary gland, optic nerve and chiasm, middle cranial fossa, internal carotid artery, cavernous sinus, the dura, pterygoid canal and nerve and cranial nerve III, IV, V1, V2, and VI [1,2]. Even though isolated sphenoid sinus lesion is rare, it's very significant clinically due to its undetectable anatomical location, ambiguous symptoms and the complications associated with injury to the above structures [5]. The main focus of this study is to present the different clinical 
manifestations and diagnostic strategies of isolated sphenoid non-invasive fungal sinusitis (SNIFS), in order to prevent delayed diagnosis and providing early management. It was conducted at King Fahad Specialist Hospital (KFSHD), and Qatif Central Hospital, Eastern region, Saudi Arabia.

\section{Method}

This is a retrospective clinical study which was conducted in the otolaryngology department of King Fahad specialist Hospital and Qatif Central Hospital, Saudi Arabia, from January 2008 to November 2019. Patients included were:

a) Isolated sphenoid non-invasive fungal sinusitis which were confirmed by computed tomography (CT) and/or magnetic resonance imaging (MRI). b) 2-intraoperative evidence of non-invasive fungal sinusitis within the sphenoid sinus.

\section{Result}

A diagnosis of isolated sphenoid fungal ball and allergic fungal rhinosinusitis had been made in 14 patients with the age ranging between 14-50 years old. 8 were males and the 6 remaining were females. The most common symptoms were headache and nasal discharge. Even though CT scan was the most important tool in the diagnosis, the conformation of the diagnosis was made either intraoperatively or by fungal culture. The management was only surgical. The only organism found was Aspergillum in these patients. Summarization of all cases found in [Table1].

Table 1: Data review of the patients with isolated sphenoid non-invasive fungal sinusitis.

\begin{tabular}{|c|c|c|c|c|c|}
\hline Patient No. & Age & Sex & Presenting Symptoms & Diagnosis & Culture Result \\
\hline 1 & 43 & Male & Post nasal discharge & Fungal ball & Aspergillum \\
\hline 2 & 25 & Male & $\begin{array}{c}\text { Nasal blockage, Chronic nasal } \\
\text { discharge }\end{array}$ & Allergic fungal sinusitis & Aspergillum \\
\hline 3 & 31 & Female & Post nasal discharge & Fungal ball & - \\
\hline 4 & 27 & Female & Occipital headache & Allergic fungal sinusitis & Aspergillum \\
\hline 5 & 40 & Male & Occipital headache + facial pain & Allergic fungal sinusitis & Aspergillum \\
\hline 6 & 39 & Male & Headache + Post nasal discharge & Fungal ball & - \\
\hline 7 & 47 & Male & Headache + Post nasal discharge & Allergic fungal sinusitis & Aspergillum \\
\hline 8 & 37 & Female & Cough + Post nasal discharge & Fungal ball & - \\
\hline 9 & 50 & Female & Allergic rhinitis symptoms + headache & Allergic fungal sinusitis & Aspergillum \\
\hline 10 & 35 & Female & Headache + Post nasal discharge & Fungal ball & - \\
\hline 11 & 15 & Male & Headache + Post nasal discharge & Allergic fungal sinusitis & Aspergillum \\
\hline 12 & 14 & Male & Asymptomatic & Fungal ball & - \\
\hline 13 & 46 & Female & Headache post nasal discharge & Fungal ball & - \\
\hline 14 & 35 & Male & headache & Fungal ball & - \\
\hline
\end{tabular}

\section{Discussion}

\section{Fungal ball}

The exact mechanism of spread of fungal infection only to sphenoid sinus is unknown. It has been hypothesized that ostial closure creates an anaerobic environment favorable for growth of Aspergillus, or that chronic sinusitis predisposes to the development of FB [2]. The clinical presentation of isolated sphenoid sinus lesions is often nonspecific and vague; thus, diagnosis may be delayed in many cases [6]. Up to date, headache refractory to the medical management is the most common symptom of isolated sphenoid sinus lesion; it presents in 70 to $90 \%$ of patients [7-9]. In this series, the most presenting feature was post-nasal discharge followed by headache and rarely cough. History and physical examination have little benefit in establishing the correct diagnosis. Imaging studies (CT scanning and/or MRI) and Nasal endoscopy are essential for a thorough evaluation and management $[8,10]$. All patient did CT scan which provided valuable information regarding the diagnosis of fungal ball and its relation to bony erosion(Figure1). MRI should only provide for patient with suspicion of invasion to surrounding structures. MRI provides more precise information regarding its relationship to the cavernous sinus, dura, optic nerve, carotid artery and pituitary gland [11]. On Nasal endoscopy the most common findings are bone thickening or sclerosis of sinus walls (Figure2). This can be secondary to the inflammatory process associated with the fungal ball and is often reversible after removing it [2,6]. Microbiology confirms fungal infection and eventually identifies fungal species. The microscopic appearance of Aspergillus on direct smear is that of a conidiophore [12]. On culture, the most frequently isolated fungus is Aspergillus fumigates. One of our fungal ball cases showed aspergillusbut the remaining 7 cases had -ve culture. The surgical management of sphenoid sinus include both endoscopic and open techniques. However, endoscopic surgery has become the fundamental approach for sphenoid sinus lesions. It can be proceeded in a trans-ethmoidal fashion or through the anterior sphenoid sinus wall directly at the site of the natural ostium [13]. It's necessary to open the sphenoid sinus and allowing complete removal of fungal debris by suction and multiple washing. Because all patients had noninvasive fungal ball no post-operative medical treatment was necessary. Post-operative follows up was done for all patient with no complication and recurrence. 


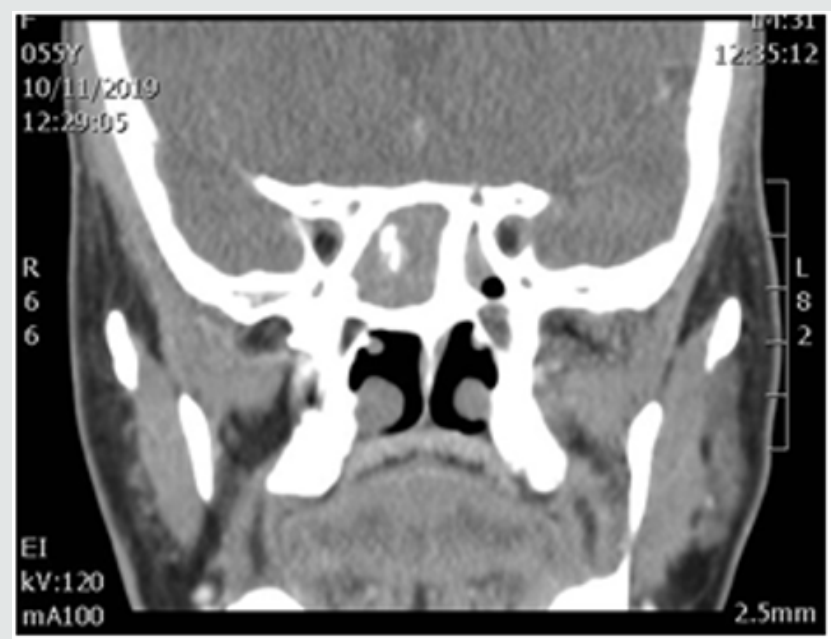

Figure 1: A computed tomography (CT) scan, coronal view of the paranasal sinuses, showing a hyperdense opacity filling the sphenoid sinus.

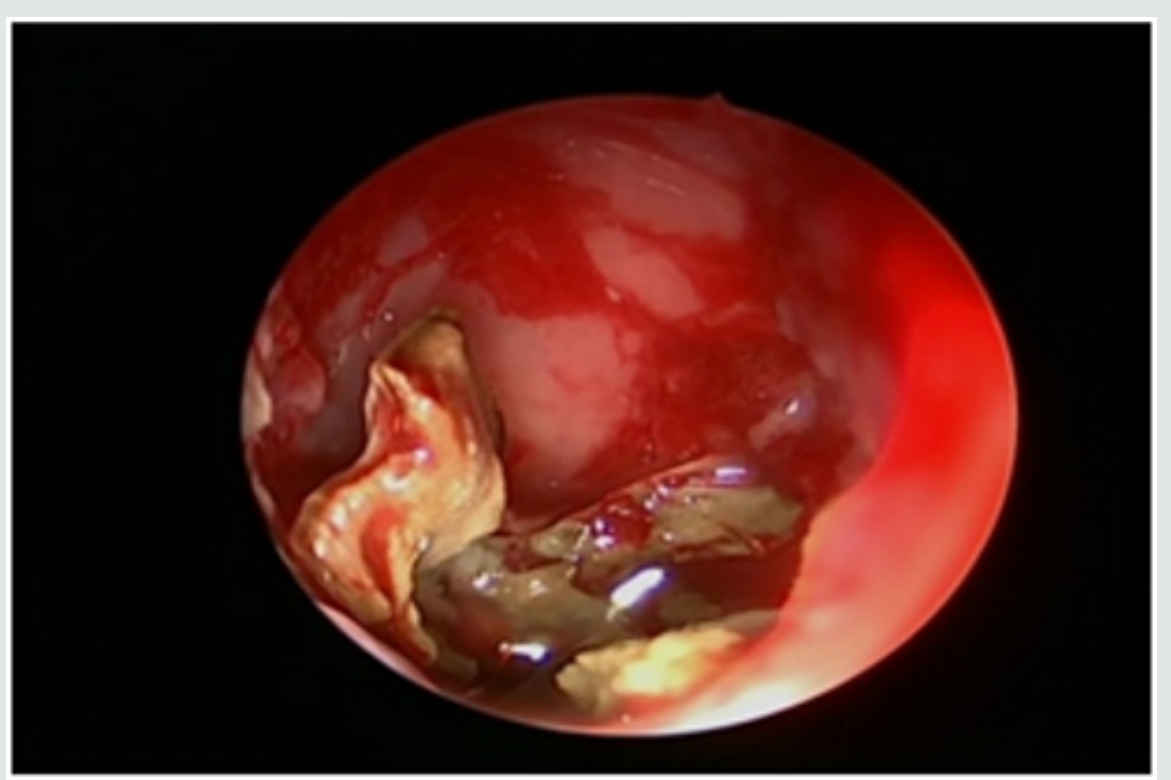

Figure 2: Endoscopic intraoperative view of The sphenoid sinus after sphenoidotomy showing Fungal material inside sinus cavity.

\section{Allergic fungal sinusitis}

The clinical presentation of SAFRS is often nonspecific and vague; thus, diagnosis may be delayed in many cases [6]. Up to date, headache refractory to the medical management is the most common symptom of isolated sphenoid sinus lesion; it presents in 70 to $90 \%$ of patients [7,9]. In this series, the most presenting feature was headache followed by nasal discharge. History and physical examination have little benefit in establishing the correct diagnosis. Imaging studies (CT scanning and/or MRI) and Nasal endoscopy are essential for a thorough evaluation and management $[8,10]$. All patient did CT scan which provided valuable information regarding the diagnosis of SAFRS and its relation to bony erosion
(Figure3). Endoscopic surgery has become the fundamental approach for SAFRS. It's includes surgical debridement of fungal material in order to open and ventilate the sinuses (Figure4), and post-operative steroid to control the recurrence. There are few reports about complication of ISAFRS. Actually, the main complication of allergic comes after sphenoid sinus involvement. The main complications are visual disturbances, diplopia and cavernous sinus thrombosis[14-15]. We have reported one case in our series with cavernous sinus thrombosis as a complication of isolated sphenoid allergic fungal sinusitis. Left sphenoidotomy was done and she was treated with Meropenem, Vancomycin, Amphotericin B and Enoxaparin and improved. 


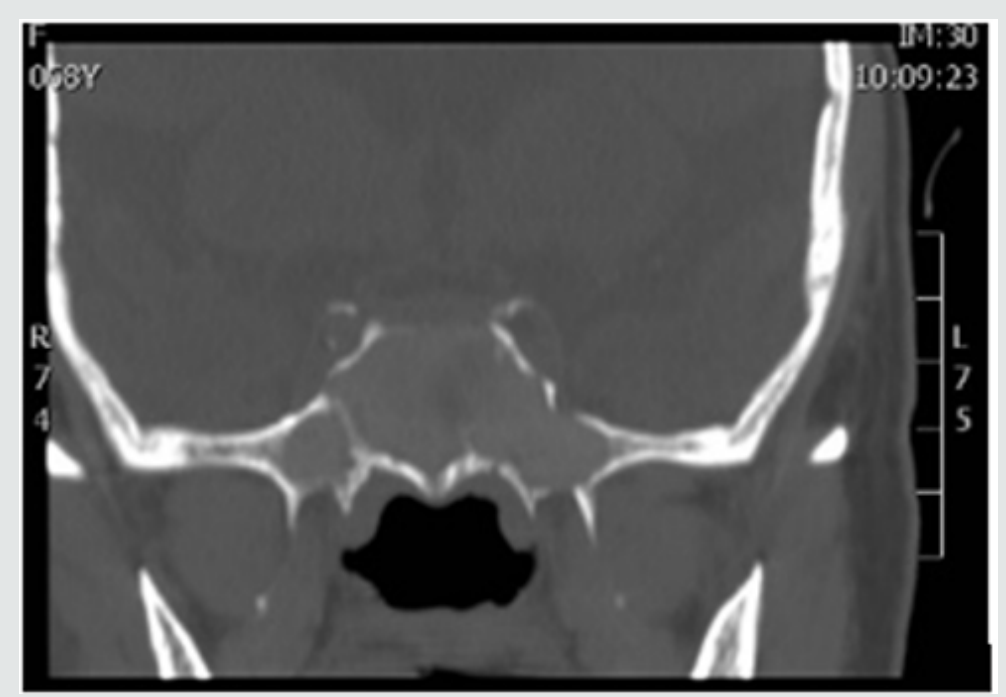

Figure 3: A computed tomography (CT) scan, coronal view showing a heterogeneous opacity filling the sphenoid sinus.

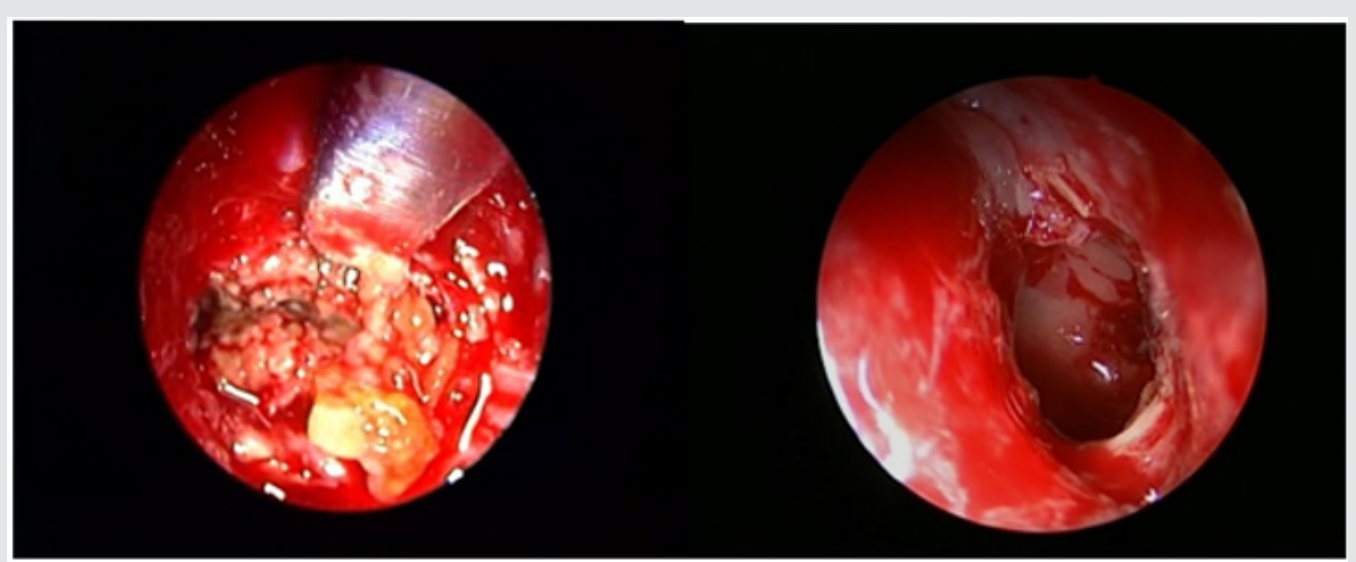

Figure 4: (A) Intraoperative endoscopic view of the allergic mucin and fungal mud filling the sphenoid sinus. (B) Endoscopic view of the Post-operative wide sphenoidotomy procedure.

\section{Conclusion}

Even though isolated sphenoid fungal ball is rare, it's clinically important because untreated SNIFS can cause significant complications. The clinical features of SNIFS are ambiguous and nonspecific which make its diagnosis more difficult. Post-nasal discharge and headache refractory to the medical management is the most common symptom of SNIFS. CT scan is still the cornerstone of radiological diagnosis of SNIFS. Endoscopic sinus surgery is the standard care of management. Post-operatively, the majority of patient had good results with no complication and recurrence.

\section{Conflict of interest}

The authors declare that there is no conflict of interest regarding the publication of this paper.

\section{Sources of funding}

There is no financial support and sponsorship.

\section{Consent}

Written informed consent was obtained from the parents for publication of this case report on behalf of the patient.

\section{References}

1. Pugliese A (2013) The multiple origins of Type 1 diabetes (2013). Diabet Med 30(2): 135-146.

2. Kiec Wilk B, Matejko B, Razny U, Stankiewicz M, Skupien J, et al. (2016) Hypoglycemic episodes are associated with inflammatory status in patients with type 1 diabetes mellitus. Atherosclerosis 251: 334-338.

3. Witte DR, Tesfaye S, Chaturvedi N, Eaton SEM, Kempler P, et al. (2005). Risk factors for cardiac autonomic neuropathy in type 1 diabetes mellitus. Diabetologia 48(1): 164-171.

4. May O, Graversen CB, Johansen MO, Arildsen H (2018) The prognostic value of the 10 frontal QRS-T angle is comparable to cardiovascular autonomic neuropathy regarding long-term mortality in people with diabetes. A population based study. Diabetes Res Clin Pract 142: 264268.

5. Herder C, Roden M, Ziegler D (2019) Novel Insights into Sensorimotor and Cardiovascular Autonomic Neuropathy from Recent-Onset Diabetes 
and Population- Based Cohorts. Trends Endocrinol Metab. 30(5): 286298.

6. Agashe S, Petak S (2018) Cardiac Autonomic Neuropathy in Diabetes Mellitus. Methodist Debakey Cardiovasc J 14(4): 251-256.

7. Pop-Busui R (2010) Cardiac autonomic neuropathy in diabetes: a clinical perspective. Diabetes Care 33(2): 434-441.

8. Cardenas-Gonzalez M, Srivastava A, Pavkovic M, Bijol V, Rennke HG, et al.(2017) Identification, Confirmation, and Replication of Novel Urinary MicroRNA Biomarkers in Lupus Nephritis and Diabetic Nephropathy. Clin Chem 63(9): 1515-1526.

9. Sebastiani G, Vendrame F, Dotta F (2011) MicroRNAs as new tools for exploring type 1 diabetes: Relevance for immunomodulation and transplantation therapy. Transplant Proc 43(1): 330-332.

10. Miao C, Chang J, Zhang G, Fang Y (2018) MicroRNAs in type 1 diabetes: new research progress and potential directions. Biochem Cell Biol 96(5): 498-506.

11. Nielsen LB, Wang C, Sorensen K, Bang-Berthelsen CH, Hansen L, et al. (2012) Circulating levels of microRNA from children with newly diagnosed type 1 diabetes and healthy controls: evidence that miR25 associates to residual beta-cell function and glycaemic control during disease progression. Exp Diabetes Res 2012: 896362.

12. Snowhite IV, Allende G, Sosenko J, Pastori RL, Messinger Cayetano S, et al. (2017) Association of serum microRNAs with islet autoimmunity, disease progression and metabolic impairment in relatives at risk of type 1 diabetes. Diabetologia 60(8): 1409-1422.

13. Xourgia E, Papazafiropoulou A, Melidonis A (2018) CirculatingmicroRNAs as biomarkers for diabetic neuropathy: A novel approach. World J Exp Med 8(3): 18-23.

14. Ciccacci C, Morganti R, Di Fusco D, D Amato C, Cacciotti L, et al.(2014) Common polymorphisms in MIR146a, MIR128a and MIR27a genes contribute to neuropathy susceptibility in type 2 diabetes. Acta Diabetol 51(4): 663-671.

15. Simeoli R, Fierabracci A (2019) Insights into the Role of MicroRNAs in the Onset and Development of Diabetic Neuropathy. Int J Mol Sci 20(18): E4627.
16. Boulton AJM, Vinik AI, Arezzo JC, Bril V, Feldman EL, et al. (2005) Diabetic neuropathies: a statement by the American Diabetes Association. Diabetes Care 28(4): 956-962.

17. Pawlinski L, Gastol J, Fiema M, Matejko B, Kiec-Wilk B (2019) Is treatment of type 1 diabetes mellitus (insulin therapy, metabolic control) optimal for preventing cardiovascular autonomic neuropathy? Endokrynol Pol 70(4): 323-329.

18. Vlachos IS, Paraskevopoulou MD, Karagkouni D, Georgakilas G, Vergoulis T, et al. (2014) DIANA-TarBase v7.0: indexing more than half a million experimentally supported miRNA:mRNA interactions. Nucl Acids Res 44(W1): W128-34.

19. Reczko M, Maragkakis M, Alexiou P, Grosse I HA (2012) Functional microRNA targets in protein coding sequences. Bioinformatics 28(6): 771-776.

20. IBM Corp (2017) IBM SPSS Statistics for Windows.

21. Pop Busui R, Boulton AJM, Feldman EL, Vera Bril, Roy Freeman, et al. (2017) Diabetic Neuropathy: A Position Statement by the American Diabetes Association. Diabetes Care 40(1): 136-154.

22. Soedamah Muthu SS, Chaturvedi N, Witte DR, Stevens LK, Porta M, et al. (2008) Relationship between risk factors and mortality in type 1 diabetic patients in Europe: the EURODIAB Prospective Complications Study (PCS). Diabetes Care 31(7): 1360-1366.

23. Li Y, Ma W, Xie C, Yin X, Fenfen Wang, et al. (2016) Identification of genes and signaling pathways associated with diabetic neuropathy using a weighted correlation network analysis A12 consort study. Med (United States) 95(47): 1-7.

24. Jiménez Lucena $R$, Rangel Zúñiga OA, Alcalá-Díaz JF, Javier López-Moreno, Irene Roncero Ramos, et al. (2018) Circulating miRNAs as Predictive Biomarkers of Type 2 Diabetes Mellitus Development in Coronary Heart Disease Patients from the CORDIOPREV Study. Mol Ther - Nucleic Acids 12: 146-157.

25. Kim JW, You YH, Jung S, H Suh Kim, IK Lee, et al. (2013) MiRNA-30a$5 p$-mediated silencing of Beta2/NeuroD expression is an important initial event of glucotoxicity-induced beta cell dysfunction in rodent models. Diabetologia 56(4): 847-855.
This work is licensed under Creative Commons Attribution 4.0 License

To Submit Your Article Click Here: Submit Article

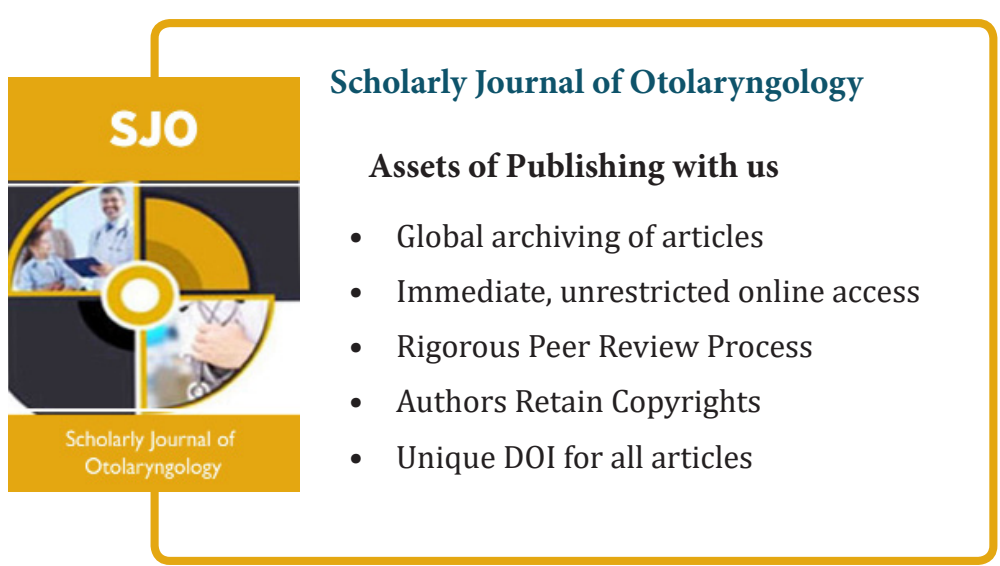

